\title{
Comment la présence de l'image influence-t-elle les décisions du traducteur? Étude des relations du couple mot/image dans la traduction filmique
}

\author{
How does the presence of image influence \\ the translator's decisions? \\ A study of the relations between words and image \\ through the example of film translation
}

\author{
Paulina Borowczyk \\ Université Adam Mickiewicz de Poznań \\ pborow@amu.edu.pl
}

\begin{abstract}
The audiovisual text constitutes an inherent and obvious whole, the components of which, being linked to each other and entering into different types of relations and interactions, contribute to the construction of its meaning. This article presents a study of cases where visual information influences decisions made by the translator and ultimately sometimes changes the translation of dubbed dialogues. In the analysis, the following examples are distinguished:

- those in which, under the influence of the image, the translator modifies the source text (compared to the original version) by adapting it to the visual contents of the audiovisual document, or by adding information to the target text;

- one in which, under the influence of the image, both the author of the original version and the translator modify one of the elements of a given idiomatic expression.

In this regard, contemporary computer-animated films (such as « Shrek 2 », « Madagascar » and « Monsters, Inc. »), aimed at all types of audiences and translated for dubbing, where the visual component plays a prominent role, represent interesting cases.
\end{abstract}

Keywords: audiovisual translation, film translation, dubbing, relations between words and image 
La réflexion et l'étude de cas choisis que nous présentons dans le cadre du présent article ne constituent qu'une petite partie d'un projet de recherche plus vaste consacré à la spécificité de la traduction audiovisuelle, et plus précisément, aux techniques de traduction des éléments culturels en fonction de la méthode de transfert (doublage vs sous-titres), ainsi qu'aux relations intertextuelles dans les films d'animation. L'observation des exemples que nous proposons n'est qu'un point de départ pour une étude plus élargie. Notre objectif est, d'un côté, de signaler l'importance des relations entre le mot et l'image, et de l'autre, d'observer comment la présence de l'image influencet-elle les décisions d'un traducteur.

\section{LE TEXTE AUDIOVISUEL}

Il est indéniable que les nouvelles technologies et la numérisation ont envahi notre quotidien. Les productions contemporaines accessibles sur tout type d'écran (poste de télévision, écran de cinéma, ordinateur, tablette, téléphone portable...) et auxquelles nous sommes confrontés chaque jour, ne sont que rarement uniquement constituées d'un texte compris comme « une chaîne linguistique parlée ou écrite formant une unité communicationnelle » (Ducrot, Schaeffer, 1995, p. 594), mais elles sont construites par des éléments de nature différente : verbaux (oral et/ou écrit), visuels (image fixe, image animée, photographies...), sonores (musique, bruits...). Le texte audiovisuel et plus précisément le texte filmique qui nous intéresse le plus dans le cadre du présent article - se divise, selon Zoë Pettit (2008), en quatre catégories principales : «1) le visuel, 2) l'auditif, 3) le verbal et 4) le non-verbal. Tout ce qui est verbal (qui peut être d'ordre visuel ou auditif) tourne autour du langage, des mots, tandis que le non-verbal (également d'ordre visuel ou auditif) porte sur le non-dit (gestualité, bruitages, effets créés par le montage etc.) » (p. 102). Étant donné la présence conjointe de plusieurs éléments dans une œuvre filmique, il est important de mettre en relief le fait que le texte audiovisuel constitue un tout dont les composantes intrinsèques, étant liées l'une à l'autre et entrant dans différents types de relations et d'interactions, contribuent à la construction du sens de celui-ci. Comme le remarque Martine Joly (2004) « les mots et images se relaient, interagissent, se complètent, s'éclairent avec une énergie vivifiante. Loin de s'exclure, les mots et les images se nourrissent et s'exaltent mutuellement» (p. 116).

Qu'en est-il pour un traducteur qui s'engage à traduire un texte filmique et le rendre accessible à un public ressortissant d'une autre communauté linguistique et culturelle ? Vu la complexité du texte filmique et le fait que «plusieurs systèmes sémiotiques entrent en jeu pour construire le sens » (Tomaszkiewicz, 1999, p. 220), le traducteur audiovisuel, contrairement au traducteur des textes écrits, doit obligatoirement prendre en considération la coexistence du verbal, du visuel et du sonore ainsi que les rapports étroits qui s'établissent entre ceux-ci lorsqu'il transfère les énoncés 
dans une autre langue. Le verbal n'est qu'une partie de tout un « complexe sémiotique » (voir Tomaszkiewicz, 2006) qu'il traduit. Il doit donc veiller à ne pas rompre dans la traduction le lien entre la couche verbale (les énoncés) et le langage corporel des protagonistes (les gestes et la mimique) qui existe dans la version originale. Comme le rappele Winkin, « la parole ne va jamais sans le geste qui la complète » (Winkin, 1981, cité dans Pettit, 2008). Il appartient donc au traducteur de vérifier si un énoncé traduit correspond à ce que fait l'acteur et à ce qui se passe à l'écran.

Comme le souligne encore la chercheuse britannique, quelle que soit la méthode du transfert audiovisuel utilisée, « (...) dans les genres audiovisuels où les particularités d'un personnage sont marquées, il est important de préserver la cohérence entre ce que «dit» l'image et ce que «dit» le discours » (Pettit, 2008, p. 104). Cette cohérence et le maintien des relations entre le verbal et le visuel dans un texte filmique traduit ont un réel impact sur la façon dont le public d'arrivée, qui conçoit le message à travers deux canaux, le visuel et l'acoustique, construira le sens. Il en découle qu'un traducteur audiovisuel ne peut se limiter qu'aux seules formes verbales. Il doit avant tout tenir compte de la nature complexe du texte audiovisuel, car celle-ci peut le diriger dans ses choix traductionnels. Brisset (2018) rappelle ainsi le statut singulier de l'adaptateur de doublage en insistant sur le fait que « les incidences sur le travail du traducteur sont donc multiples, car il doit recréer un sens filmique au-delà du simple texte verbal, en s'approchant au plus près de la cohésion intersémiotique initiale » (p. 36).

Dans les films doublés qui seront examinés dans ce qui suit, aussi bien les gestes et la mimique que les mouvements des lèvres des personnages doivent être synchronisés avec le discours.

\section{SPÉCIFICITÉ DU DOUBLAGE ET SKOPOS}

Le doublage, l'une des méthodes de traduction audiovisuelle pour laquelle optent des pays tels que l'Allemagne, l'Italie, la France ou la Hongrie, consiste à remplacer « la piste originale des dialogues en langue source d'un film (ou de tout texte audiovisuel) est remplacée par une autre piste sur laquelle des dialogues traduits ont été enregistrés dans la langue cible. Les pistes restantes restent intactes (la bande-son - y compris la musique et les effets spéciaux - et les images » (Chaume, 2012, p. 1 ; notre traduction). Cette forme de traduction, où les voix originales des acteurs sont remplacées par des voix d'autres acteurs s'exprimant dans une autre langue, est soumise à des contraintes et exigences techniques spécifiques et, lors de sa réalisation, fait appel à plusieurs métiers particuliers, comme p.ex. le directeur de doublage, le détecteur, l'adaptateur dialoguiste, l'ingénieur du son, les comédiens... Le procédé technique comprend alors plusieurs étapes. Sans entrer dans les détails de toutes les étapes du processus du doublage, le présent article se concentre surtout sur celles qui sont liées au respect des mouvements des lèvres et des gestes des comédiens pendant l'acte traduisant. 
Tout d'abord, lors de la phase de détection, le technicien s'occupe du « repérage image par image du mouvement des lèvres de chaque personnage et [de la] transcription des dialogues originaux sur la bande rythmo » (Le Nouvel, 2007, p. 12). De plus, le détecteur doit indiquer les rires, les réactions des acteurs, le débit verbal, les respirations, les pauses, ainsi que les changements de plan. Cette phase, pendant laquelle on fait une véritable analyse phonétique et restitue, de manière très exacte et rigoureuse, les ouvertures et fermetures de bouche des personnages à l'écran, sera d'une grande aide pour l'adaptateur dialoguiste qui, à son tour, « transpose, sur la bande rythmo détectée, les dialogues de la langue source dans la langue cible, en tenant compte des référents culturels et du mouvement des lèvres » (Le Nouvel, 2007, p. 12). En s'inspirant des signes de détection inscrits par le détecteur, l'adaptateur tente de les replacer aux mêmes endroits dans sa traduction (on parle de synchronisation labiale). En outre, il doit prendre en compte toutes les informations fournies par l'image, c'est à dire le langage corporel des acteurs. Robert Paquin (2010), adaptateur anglais-français de films et de téléséries, met en relief l'importance de la synchronisation dans le métier d'adaptateur traducteur : " étant donné qu'on travaille avec une image, ce qui nous concerne avant tout, ce sont les sons qu'on peut voir, surtout ceux qui nécessitent une fermeture de la bouche. L'art de la traduction/adaptation pour le doublage synchrone se résume essentiellement à un seul terme : synchronisation » (p. 7). Pourtant, le synchronisme phonétique n'est pas le seul qu'il faille respecter car, comme le remarque Paquin (2010), il faut prendre en considération le rapport entre ce qui est dit et l'image. « Par exemple, si le personnage à l'écran dit : « There are a thousand good reasons to.... », l'adaptateur écrira peut-être : «Il y a des centaines de raisons qui justifient...», pour éviter de mettre « mille », avec son « $\mathrm{m}$ » bilabial, surtout si c'est un gros plan et qu'on voit bien la bouche. Mais si le personnage dit « three thousand » et écrit au tableau « 3000 », l'adaptateur n'a parfois pas le choix de sacrifier le synchronisme phonétique au profit du synchronisme sémantique, puisque le spectateur voit le chiffre à l'écran » (p. 7). De nos jours, dans les studios de doublage, la détection et l'adaptation synchrones des dialogues se font à l'aide de logiciels et systèmes informatiques, comme p.ex. Synchronos ou DubStudio.

Une fois la traduction finie, c'est en présence du directeur artistique et de l'ingénieur du son que l'on procède à l'enregistrement des voix des comédiens en langue d'arrivée. Ces derniers, suivant la bande rythmo, lisent leur rôle, en le synchronisant du mieux possible au mouvement des lèvres, à la mimique, aux gestes, bref, au jeu des personnages à l'image. Finalement, sous la supervision du directeur de doublage « se fait le mixage définitif de la version doublée, où les nouveaux dialogues sont incorporés à la version internationale. L'ingénieur du son vérifie le synchronisme de l'enregistrement et l'améliore, si besoin est, en décalant les voix ou en jouant sur leur vitesse de défilement » (Le Nouvel, 2007, p. 11). On peut donc bien voir que dans le doublage, ce sont aussi bien l'image que le son qui guident l'adaptateur dans ses choix pendant la traduction. 
Une autre particularité du doublage, procurée par la disparition du discours original et soulevée par les chercheurs et les traducteurs audiovisuels (à ce propos voir Lang, 1996 ; Lambert \& Delabastita, 1996 ; Ramière, 2004 ; Le Nouvel, 2007 ; Chaume, 2012 ; Sikora, 2013 ; Cornu, 2014 ; Plewa, 2015 ; Brisset, 2017), est liée au fait que dans cette méthode de traduction audiovisuelle le traducteur dialoguiste dispose de plus de liberté qu'un sous-titreur, par exemple. Étant donné que la bande sonore originale (les dialogues des protagonistes) est entièrement remplacée par la bande sonore contenant les dialogues formulés en langue d'arrivée, les spectateurs de la version traduite n'ont aucun accès au discours étranger et par conséquent ne peuvent pas se permettre de comparer la V.O. avec sa traduction, comme c'est le cas du sous-titrage où l'original est audible. De manière générale, partant du fait que le doubleur a une plus grande marge de manœuvre que le sous-titreur on remarque en Pologne une certaine tendance, et ce à partir de 2001 et la version polonaise de Shrek, à "domestiquer" la traduction des films d'animation : c'est à partir de ce moment-là, comme le souligne Plewa (2015), les doubleurs effectuent une traduction saturée de références à la réalité et à la culture polonaise (2015, p. 13).

Les témoignages qui suivent montrent que la disparition du dialogue initial peut provoquer l'effacement du caractère étranger de l'original et que l'adaptateur vise à créer une version langagière adaptée aux attentes de l'audience cible.

- « Dans l'opération de doublage, le scénario traduit est soumis à une adaptation, qui permet d'en modifier le texte pour synchroniser la nouvelle bandeson avec l'image. Ce qu'entend le spectateur à la projection du film en version doublée (VD) est une version retravaillée du scénario traduit, qui, même si elle présente de nombreux points communs avec celui-ci, en diverge nettement sur d'autres » (Brisset, 2017, p. 33).

- « Le doublage est dès lors un authentique discours de substitution, et il tend souvent à s'instaurer comme le seul discours, au point de faire oublier l'étranger et l'exotisme. C'est par ailleurs sa raison d'être : il remplace en effet une langue étrangère et tend à la faire oublier » (Lambert \& Delabastita, 1996, p. 54).

- [Dans le doublage] « il s'agit de recréer toute la bande sonore du film pour créer l'illusion d'une action qui se déroule dans la langue cible. Le doublage illustre bien la complexité des interventions nécessaires pour créer l'illusion de la transparence » (Amzallag, 2010, p 3).

- «Dans le doublage, le traducteur peut prendre beaucoup plus de libertés dans la mesure où l'original disparaît. Cependant, il est tout aussi indispensable de coller au rythme de l'écriture, de la mise en scène et du montage, ainsi qu'à la gestuelle et au jeu des acteurs. Et, bien sûr, au sens ! C'est un travail qui demande aussi un peu plus de temps, car dès qu'un personnage remue les lèvres, il faut introduire du texte. Cette liberté est toutefois soumise, en effet, à l'impératif du synchronisme » (Meininger, 2013, p. 13). 
- «Translation for dubbing really requires adaptation. It's not just a question of translating dialogues, they have to be rewritten » (Paquin, 1998, cité dans Brisset, 2017).

Que peut-on conclure de ce qui précède ? Que le doublage n'est pas tant une traduction au niveau des dialogues qu'une adaptation du discours « en raison des contraintes sous lesquelles ces opérations [les auteurs parlent du sous-titrage et du doublage] sont souvent effectuées » (Lambert \& Delabastita, 1996, p. 35). Deuxièmement, imposées par la matière audiovisuelle ces limitations font en sorte que l'adaptation ne puisse pas être « un calque de la bande originale, une traduction littérale du texte, mais une réécriture, une variation tout autant qu'une interprétation. C'est même la raison pour laquelle les adaptateurs sont considérés comme des auteurs et touchent les droits afférents à ce statut » (Le Nouvel, 2007, p. 43). Et troisièmement, dans les ouvrages traitant de la traduction audiovisuelle, le doublage est perçu comme une méthode marquée par la visée cibliste et la stratégie de naturalisation et d'adaptation (voir entre autres Danan, 1991 ; Ramière, 2004 ; Sikora, 2013 ; Brisset, 2017). Une telle approche envers la traduction du texte filmique fait penser à la théorie du skopos (Skopostheorie), qui est fondée sur les finalités communicatives d'un texte à traduire. Selon cette approche fonctionnaliste, créée par Katharina Reiss et Hans Josef Vermeer (1984) puis nettement développée par Christiane Nord (1997) la traduction est une activité ciblée où il s'agit de la transmission du but/de la finalité du texte de départ, et non pas de la simple reproduction des mots/des phrases. Le but principal de la traduction consiste à " susciter chez les destinataires du texte cible une réaction identique à celle des destinataires du texte source, même si pour ce faire il faudra modifier le contenu ou des éléments stylistiques du texte source » (Nord, 2008, p. 53). Le but de la version originale du film et celui de la version doublée en une autre langue peut être le même ou tout à fait différent. C'est donc au traducteur de décider si la version originale du film ne va être qu'un point de départ soit pour créer une adaptation, soit pour restituer fidèlement chaque élément. «Cela siginfie qu'un même texte peut avoir plusieurs traductions acceptables parce que chacune répond à un skopos particulier » (Guidère, 2010, p. 73).

Comment toutes ces remarques se transposent-elles à la traduction au doublage des films d'animation? Même si l'adaptateur est obligé de se soumettre à des contraintes techniques, il profite d'une liberté d'auteur considérable en raison de la disparition des dialogues originaux. Par conséquent, dans les films d'animation à effet ludique et faisant référence à un monde inventé et fabuleux, le traducteur peut se permettre d'introduire des modifications d'ordre lexical, sémantique, voire culturel dans la version doublée, dans le but de déclencher un effet de communication similaire à celui de l'original (l'effet comique). Ce qui nous intéresse le plus, ce sont les rapports entre l'image et le son, ainsi que les éventuelles divergences entre la V.O. et la version doublée en polonais. Quelles sont les conséquences d'une telle liberté pour un adaptateur dialoguiste face à la synchronisation de l'image avec les énoncés des personnages ? 


\section{ÉTUDE DE CAS : LE DOUBLAGE DANS LES FILMS D’ANIMATION}

Dans le cadre du présent article, nous nous pencherons sur des questions telles que : l'adaptateur s'appuie-t-il essentiellement sur les données linguistiques, ou prend-il également en considération la couche visuelle ? Comment la présence de l'image influence-t-elle la traduction de la parole ? Sur quelle base le traducteur introduit-il des modifications dans le texte d'arrivée par rapport à celles contenues dans la V.O. ? Nous tenterons ainsi de voir le lien entre l'image et les énoncés en anglais et doublés en polonais.

Pour donner suite aux réflexions théoriques qui précèdent, il est surtout intéressant d'étudier les cas où les informations visuelles influencent les décisions prises par le traducteur et peuvent jouer sur le doublage des dialogues. Nous allons observer dans quelle mesure le traducteur polonais a effectivement tenu compte des informations significatives à l'écran et si ses choix de traduction ont été dictés par la présence de l'image et sous son influence. C'est sur cette interaction entre l'image et les dialogues - original et traduit - que nous nous penchons dans les exemples qui suivent.

Il ressort de l'analyse que dans certains cas, sous l'influence de l'image, le traducteur modifie le texte d'arrivée (par rapport à la V.O.) en l'adaptant à la couche visuelle (exemples 1 et 2 ) ou bien ajoute au texte d'arrivée des informations absentes de l'original (exemples 3 et 4) ; tandis que dans d'autres, sous l'influence du contenu visuel, aussi bien l'auteur de la V.O. que le traducteur-dialoguiste modifient l'un des éléments d'une expression idiomatique (exemple 5).

À cet égard, les exemples tirés de films d'animation contemporains («Shrek 2 », « Madagascar » et « Monstres et Compagnie ») destinés à tout type de public, traduits de l'anglais en polonais à l'aide du doublage et dans lesquels la composante visuelle joue un rôle de premier plan, seront examinés en fonction des modifications introduites dans la traduction sous l'influence de ce qui passe à l'écran.

Pour présenter le mieux possible les exemples illustrant la problématique discutée, nous fournirons ici une capture d'écran de la scène analysée ainsi que le dialogue qui l'accompagne. Les dialogues sont regroupés dans des tableaux à deux colonnes - la colonne de gauche étant réservée au texte de départ (TD) et la colonne de droite au texte d'arrivée (TA). Dans les répliques des personnages, nous soulignerons par ailleurs les fragments qui sont accentués et synchronisés à ce qui est visible dans la capture d'écran (gestes ou autres informations présentes à l'image). L'avantage de cette méthode de présentation du matériel est la possibilité d'observer la fusion de l'image avec la parole.

Dans les deux premiers cas, l'adaptateur, profitant de la présence de l'image, modifie le contenu du dialogue dans la version polonaise. Ainsi, il adapte le texte cible à l'information que les spectateurs voient à l'écran. Les deux premiers exemples ont été tirés de la production américaine «Shrek 2 » et font référence à ce qui a été discuté 
plus haut : la traduction du verbal est soumise à ce qui passe à l'écran et le traducteur tente de maintenir la cohérence entre les deux canaux.

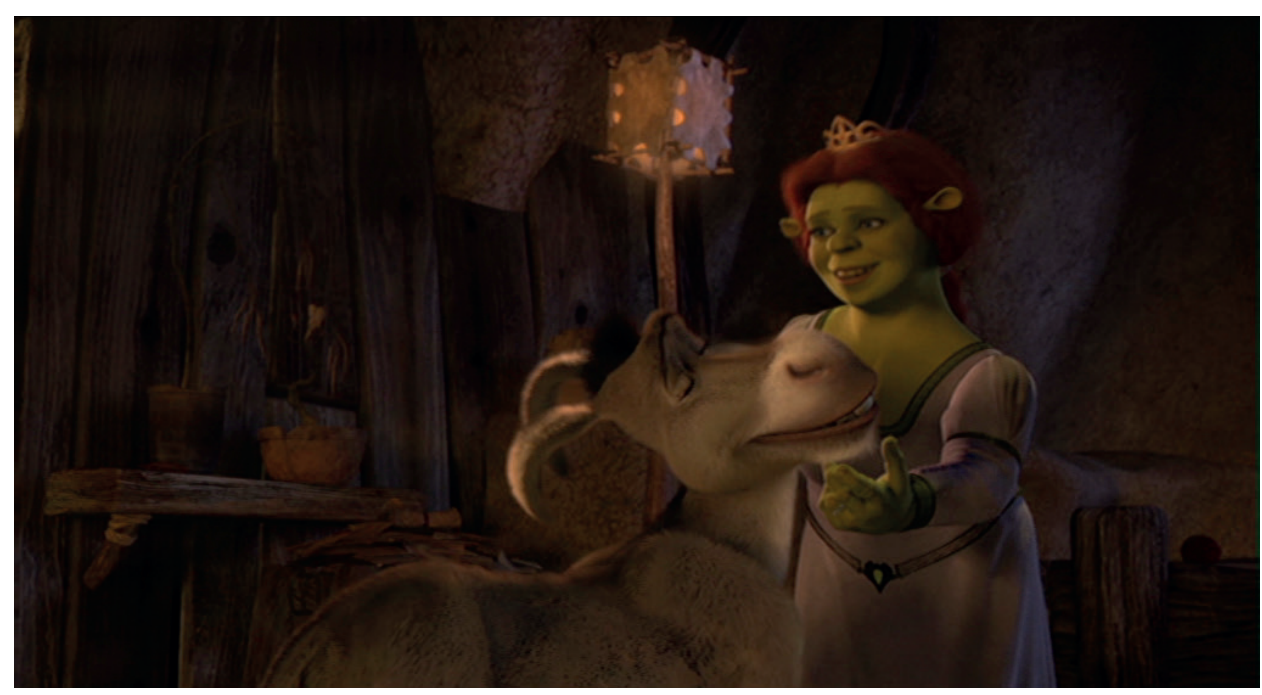

Exemple 1. Shrek 2 (2004), direction : Andrew Adamson, Conrad Vernon, Kelly Asbury

\begin{tabular}{|l|l|}
\hline \multicolumn{1}{|c|}{ Texte anglais (TD) } & \multicolumn{1}{|c|}{ Texte polonais (TA) } \\
\hline L'Âne: & L'Âne: \\
Shrek! Fiona! Aren't you two a sight for sore eyes! & Shrek! Fiona! No niech was wyściskam jak raz. \\
$\begin{array}{l}\text { Give us a hug, Shrek, |you old love machine.And } \\
\text { look at you, Mrs. Shrek. How bout a side of sugar } \\
\text { for the steed? }\end{array}$ & $\begin{array}{l}\text { Dawaj pyska, Shrek. Ty ślicznoto. A tu proszę: pani } \\
\text { Shrek. No pomiziaj mnie pod broda. }\end{array}$ \\
\hline
\end{tabular}

La capture d'écran présente une scène de salutation entre l'Âne et la princesse Fiona et Shrek, qui viennent de revenir de leur voyage de noces. L'Âne attend ses amis dans la maison des jeunes mariés. En observant les deux canaux (visuel et acoustique) à travers lesquels les informations parviennent au public, on peut constater que le traducteur a modifié l'énoncé de l'Âne dans le doublage polonais, en se référant directement au geste fait par Fiona (gratouilles sous le menton) : « No pomiziaj mnie pod brodą » (fr. Gratouille-moi sous le menton). La question qui apparaît dans le texte de départ («How 'bout a side of sugar for the steed ? ») peut être comprise de deux manières. En utilisant l'expression « a side of sugar » l'Âne demande un peu de sucre à la princesse Fiona (sens propre) ou bien, au sens figuré, il lui demande quelque chose d'agréable, comme une caresse ou un câlin. Même si dans la traduction polonaise cette ambiguité est effacée et le discours s'en trouve modifié, le message verbal correspond tout à fait à ce qui passe à l'image. La cohérence originale de l'image et du son est ainsi maintenue dans la version doublée. 


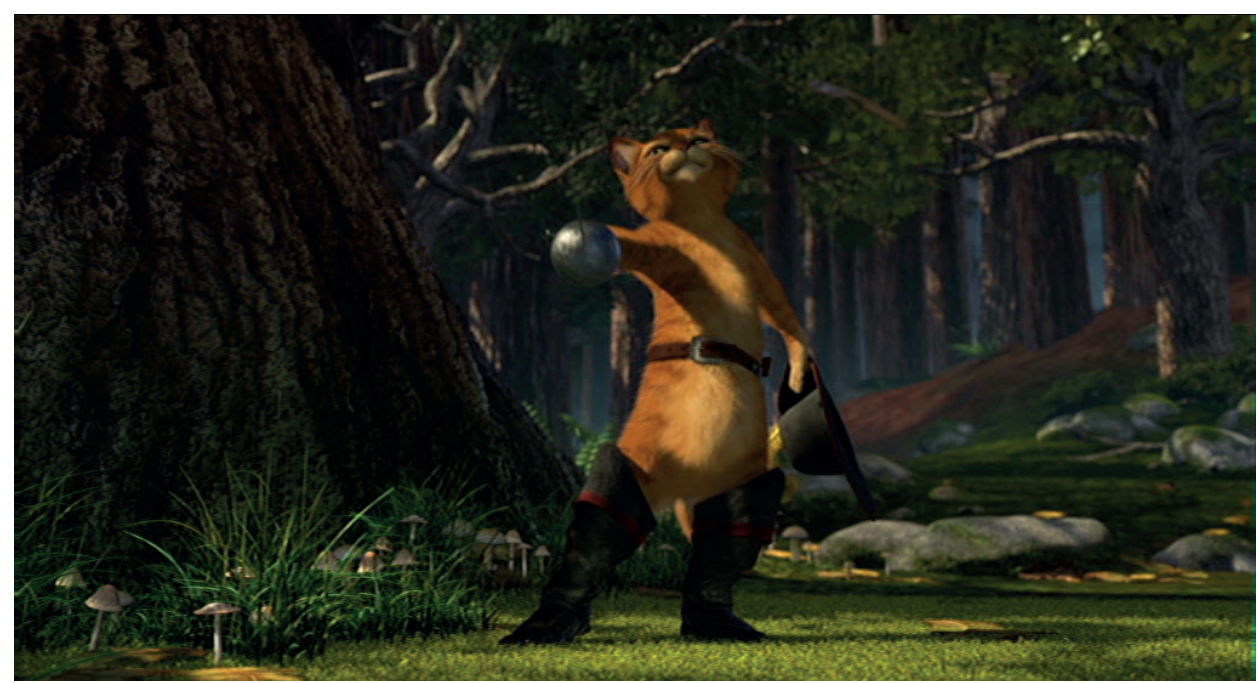

Exemple 2. Shrek 2 (2004), direction : Andrew Adamson, Conrad Vernon, Kelly Asbury

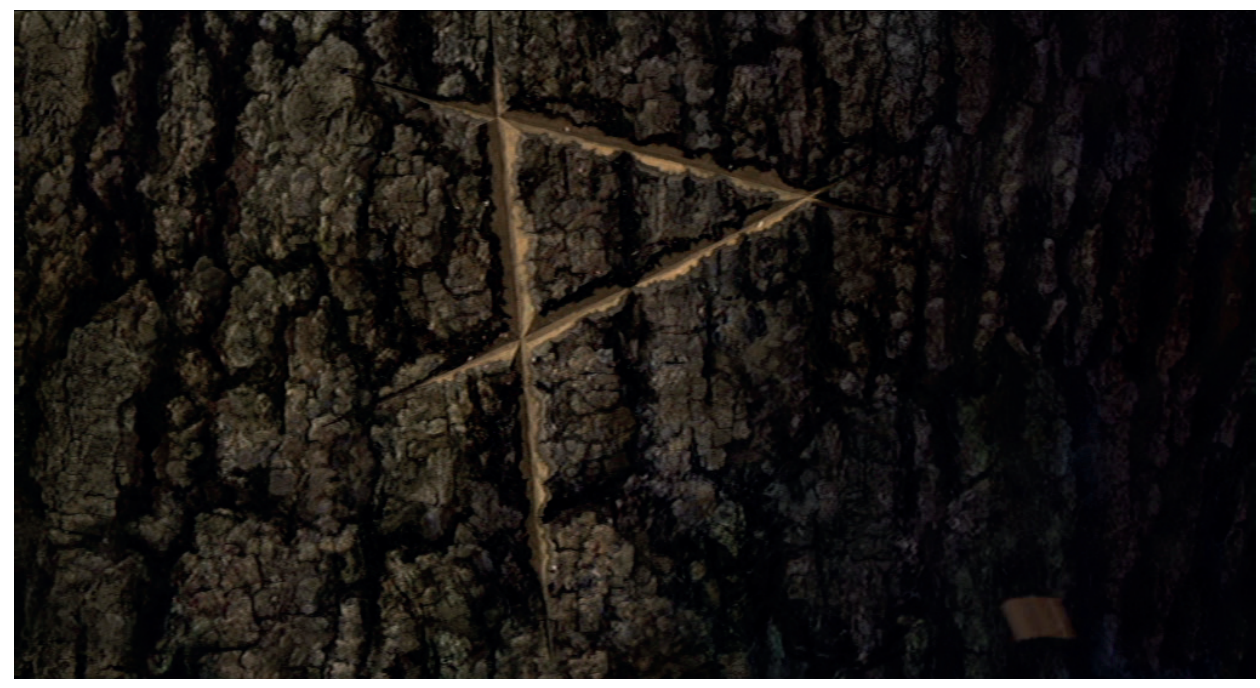

\begin{tabular}{|l|l|}
\hline \multicolumn{1}{|c|}{ Texte anglais (TD) } & \multicolumn{1}{|c|}{ Texte polonais (TA) } \\
\hline $\begin{array}{l}\text { Le Chat Potté : } \\
\text { Now,ye ogre, pray for marcy... Puss... in Boots! }\end{array}$ & $\begin{array}{l}\text { Le Chat Potté : } \\
\text { Jam Puszek Okruszek. Kto ze mna zadrze, umart } \\
\text { w butach! }\end{array}$ \\
\hline
\end{tabular}

Cet exemple constitue un vrai défi pour le traducteur dialoguiste. La première capture d'écran montre le personnage provenant du conte de Charles Perrault, le Chat botté (l'orthographe du nom change dans la version française du film en Chat potté). C'est le moment de la première rencontre du Chat potté avec Shrek et l'Âne. Afin de 
faire peur à ces derniers, le Chat potté, lorsqu'il prononce les paroles citées ci-dessus, dégaine son épée et trace la lettre $\mathrm{P}$ sur un tronc d'arbre à l'instar de Zorro (deuxième capture d'écran). C'est la première lettre du nom du héros en anglais : Puss in Boots. L'adaptateur polonais, Bartosz Wierzbięta, au lieu de recourir à l'appellation équivalente du Chat botté (en polonais : Kot w butach), dans laquelle il n'y a pas de lettre pà l'initiale des mots, a décidé de citer le nom du chien Puszek Okruszek, qui commence par la lettre P. Ce nom est connu grâce à une chanson pour enfants interprétée par la chanteuse polonaise Natalia Kukulska. En optant pour cette adaptation, le traducteur a modifié le discours traduit, mais est ainsi resté conforme aux images filmiques (voir Borowczyk, 2008). En outre, en introduisant un élément connu du public d'arrivée, il a obtenu l'effet comique attendu. Le but communicationnel, le principal postulat de la théorie du skopos, a donc été atteint.

Dans le cas suivant, le traducteur dialoguiste ajoute à la version polonaise des informations supplémentaires qui sont absentes de la V.O. L'exemple ci-dessous provient du film d'animation américain « Madagascar».

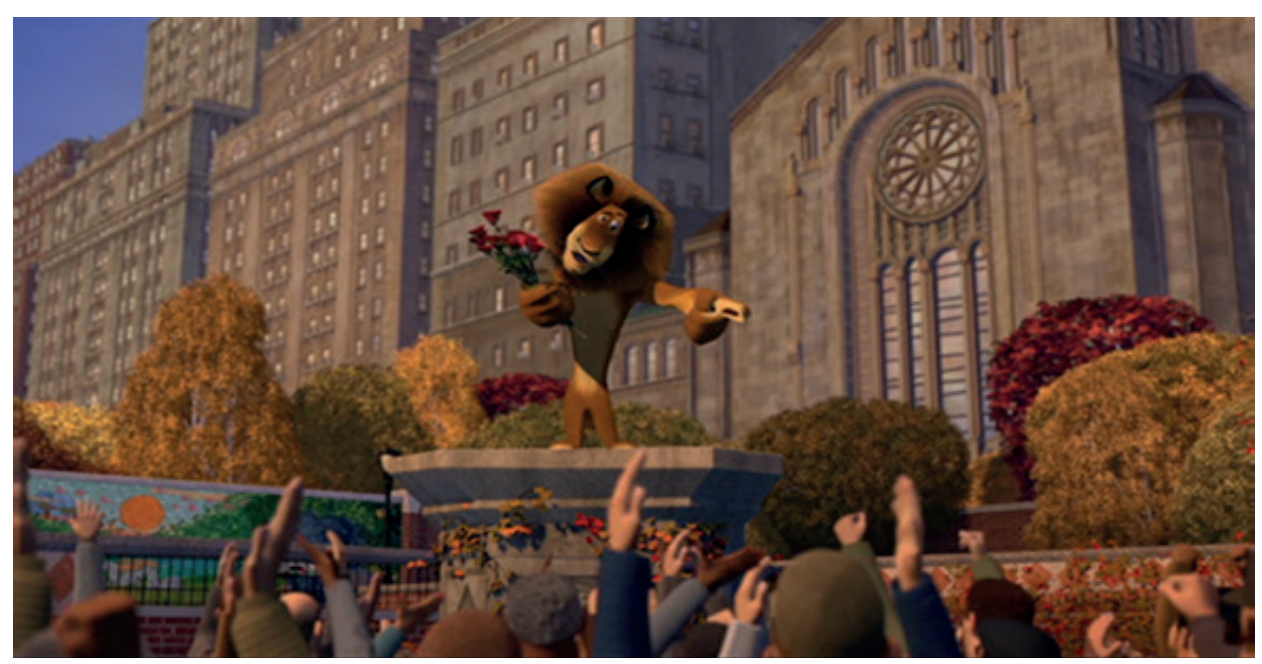

Exemple 3. Madagaskar (2005), direction : Tom McGrath, Eric Darnell

\begin{tabular}{|l|l|}
\hline \multicolumn{1}{|c|}{ Texte anglais(TD) } & \multicolumn{1}{c|}{ Texte polonais (TA) } \\
\hline Alex : & Alex : \\
You're a great crowd. & Jesteście świetni. O, pani szczególnie. \\
\hline
\end{tabular}

Alex le lion, est, aux côtés de Marty le zèbre, Melman la girafe et Gloria l'hippopotame, l'un des principaux personnages du film « Madagascar ». Ces animaux vivent au zoo de Central Park à New York, où ils mènent une vie tranquille. Dans la capture d'écran (Exemple 3), on voit Alex faire un show devant le public réuni. Chaque jour 
à la même heure, le lion joue la vedette pour les visiteurs. À la fin du spectacle, lors des applaudissements, Alex remercie le public en prononçant les paroles suivantes : «You're a great crowd! » (fr. Vous êtes un public magnifique !). Sous l'effet du geste fait par Alex (tendant l'index pour désigner une personne en particulier) l'adaptateur polonais a ajouté une information qui manquait dans le texte de départ, à savoir : « $\mathrm{O}$, pani szczególnie » (fr. Et vous tout particulièrement). L'élément ajouté est en pleine synchronie avec le geste déictique à l'image. Il est important de souligner que dans ce cas, l'image a eu une réelle influence sur la décision prise par le traducteur polonais.

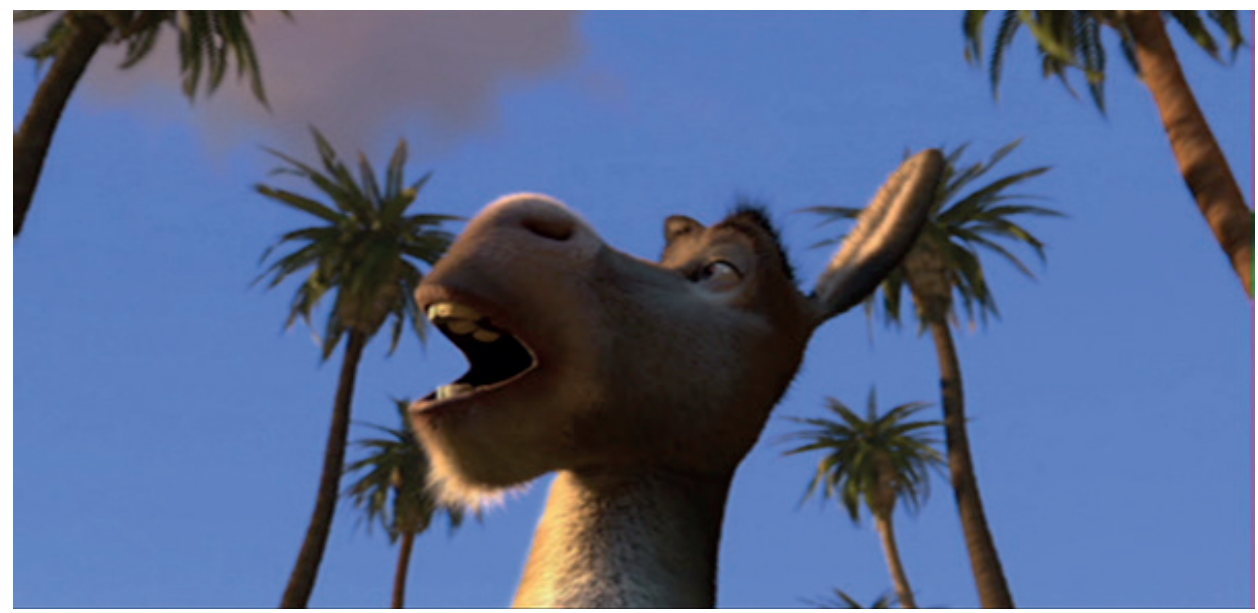

Exemple 4. Shrek 2 (2004), direction : Andrew Adamson, Conrad Vernon, Kelly Asbury

\begin{tabular}{|l|l|}
\hline \multicolumn{1}{|c|}{ Texte anglais (TD) } & \multicolumn{1}{c|}{ Texte polonais (TA) } \\
\hline L'Âne : & L'Âne : \\
Wow! & Sadzonki! \\
\hline
\end{tabular}

Dans la scène de l'exemple 4 on voit l'Âne et ses amis récemment mariés (Fiona et Shrek) tous trois invités au royaume de Fort Fort Lointain par les parents de Fiona, le roi Harold et la reine Lilian. Après un long voyage les invités arrivent enfin en ville. Impressionné par la vue magnifique et les palmiers, l'Âne, regardant tout autour de lui, se sert dans la V.O. de l'interjection «wow » pour exprimer son admiration. Cependant, l'adaptateur polonais, au lieu de calquer la même interjection, dont l'emprunt à l'anglais est entré dans l'usage en Pologne, opte pour le nom « sadzonki» (fr. les boutures) faisant référence aux palmiers visibles à l'écran. Le fait d'employer le substantif « les boutures » pour appeler les palmiers renforce l'effet comique dans le doublage polonais.

Une autre technique intéressante, dont se servent aussi bien l'auteur des dialogues originaux que le traducteur dialoguiste polonais, consiste à modifier une expression idiomatique, à cause des informations contenues dans l'image. Ils mettent ainsi le vi- 
suel à profit pour renforcer l'effet humoristique de la scène. En voici un exemple, tiré du film d'animation américain « Monstres et Compagnie» réalisé par les studios Pixar.

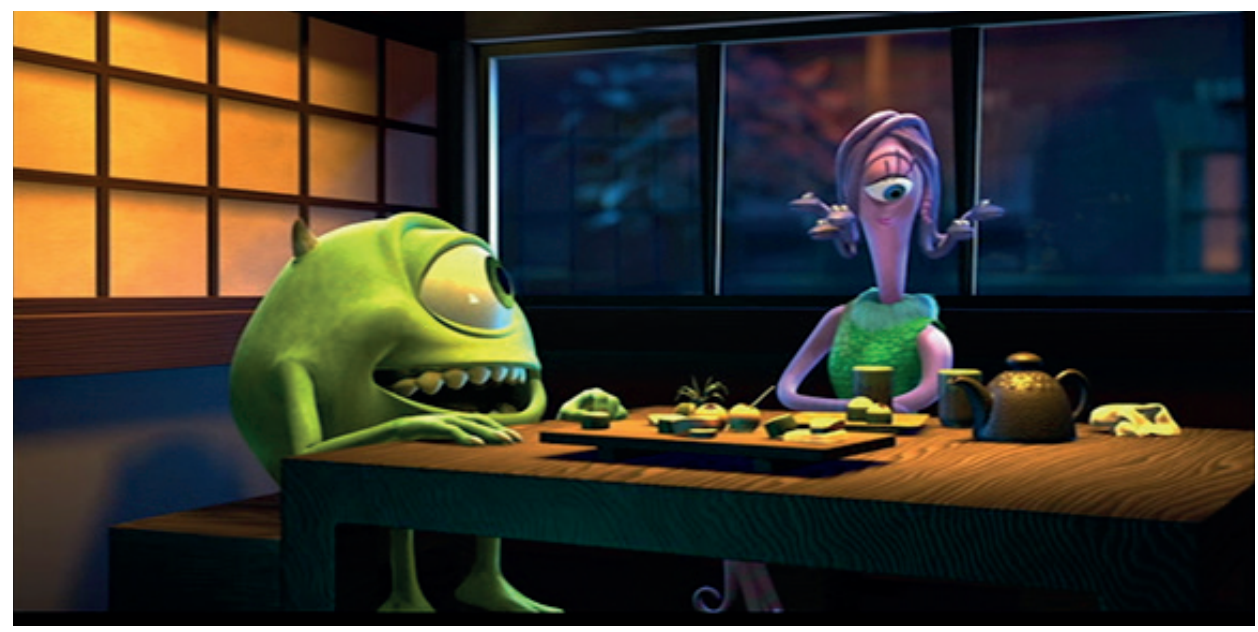

Exemple 5. Monstres et Compagnie (2002), direction : Pete Docter, David Silverman, Lee Unkrich

\begin{tabular}{|l|l|}
\hline \multicolumn{1}{|c|}{ Texte anglais (TD) } & \multicolumn{1}{c|}{ Texte polonais (TA) } \\
\hline $\begin{array}{l}\text { Mike: } \\
\text { Oh, I was just thinking about the first time I laid } \\
\text { eye on you, how pretty you looked. }\end{array}$ & $\begin{array}{l}\text { Mike: } \\
\text { Odkad cię poznatem, oka nie moge od ciebie } \\
\text { oderwać. Piękna jesteś. }\end{array}$ \\
\hline
\end{tabular}

L'histoire du film se déroule à Monstropolis, une ville habitée par toutes sortes de monstres et créatures. La capture d'écran présente deux personnages : Mike Wazowski et la secrétaire Célia lors d'un rendez-vous galant dans un restaurant. Ils sont très amoureux l'un de l'autre. Comme on le voit à l'image, ces créatures ne possèdent qu'un œil. Dans la V.O., l'auteur a utilisé l'expression idiomatique lay (one’s) eyes on someone/something, dont le sens peut être également rendu par un idiotisme en polonais tel que nie móc oderwać oczu od kogoś/czegoś. Du fait de ce que l'on voit à l'écran, la modification de l'expression consiste dans le remplacement du substantif au pluriel (ang. eyes et pol. oczu) par le substantif au singulier (ang. eye et pol. oka). Le fait de se référer à la couche visuelle se reflète ainsi dans le verbal et prouve que les adaptateurs tiennent compte d'autres éléments signifiants de l'ensemble sémiotique et pas seulement du discours.

Ces quelques exemples montrent que le traducteur audiovisuel traduit les données linguistiques toujours en référence aux informations présentes à l'image, car celles-ci peuvent influencer la manière dont le discours est traduit et dont les spectateurs en construiront le sens. Tout comme les contraintes techniques, les rapports étroits entre les canaux déterminent donc de manière considérable les décisions d'un traducteur dialoguiste. 


\section{CONCLUSION}

Comme nous l'avons mis en relief à maintes reprises dans notre article, le texte audiovisuel est, comme son nom l'indique, accompagné de plusieurs composants signifiants, telles que le texte, l'image, la musique et les bruits qui entretiennent toute sorte de relations pour véhiculer ensemble le sens. Pour rendre le sens du texte filmique dans une autre langue, le traducteur doit prendre en considération le tout sémiotique et les interactions entre les éléments mentionnés. « Entre texte écrit et texte oral, texte figé sur la page et texte joué, texte verbal et multimodalité filmique, entre auteur et spectateur, le traducteur de doublage se situe dans l'intermédiation. Son rapport au texte est multiple, tant dans la phase de réception que de production. (...) Son texte doit être fonctionnel (...), se fond dans un contexte multimodal marqué par la temporalité et repose sur un support animé et plurisémiotique » (Brisset, 2018, p. 41).

En plus, on a démontré que le doublage, considéré comme une sorte d'adaptation, est conditionné par des contraintes techniques qui influencent réellement le produit final de l'opération de traduction. Il ne s'agit pas simplement d'effectuer une bonne traduction, qui exprime le sens des dialogues tout en étant naturelle, mais pour que l'illusion soit créée il faut garantir une traduction synchrone avec les mouvements des lèvres des acteurs et correspondante à ce qui se passe à l'écran. C'est donc au traducteur dialoguiste de garder la cohérence entre l'image (les gestes, la mimique, ainsi que toute sorte d'information visuelle significative) et les paroles des protagonistes.

Sans vouloir tirer des conclusions générales, nous tenons à signaler que les exemples retenus pour notre analyse ont prouvé que les choix traductologiques de l'adaptateur étaient faits en rapport avec le contenu visuel. C'est en référence aux informations de l'image que le traducteur dialoguiste a préparé son texte d'arrivée. Parfois, le traducteur a modifié le texte d'arrivée en l'adaptant à ce qu'il y avait à l'écran (exemples 1 et 2) ou bien il a ajouté des informations absentes de l'original (exemples 3 et 4). On a aussi relevé un cas où les auteurs de la V.O. et de la version traduite ont introduit des modifications dans une expression idiomatique pour rester conforme à l'image (exemple 5). Nous pouvons observer que dans les textes doublés trois différentes stratégies ont été employées, à savoir la modification (1 et 2), l'ajout (3 et 4) et la vérification (5). Vu que dans le dernier exemple la transformation linguistique observée a été faite par les auteurs du document source et par le traducteur, l'impact de l'image dans le processus de traduction à proprement parler a été en quelque sorte atténué. Nous voudrions souligner qu'il serait souhaitable de mener des recherches plus approfondies pour pouvoir observer dans quelles situations les doubleurs adaptent le contenu textuel à l'écran et comment ces deux couches interagissent. 


\section{BIBLIOGRAPHIE}

Amzallag, Y. (2010). Dans les coulisses de la transparence : Circuit. Le doublage, l'art de l'illusion, 107, 3. Boillat, A., Cordonier, L. (2013). La traduction audiovisuelle : contraintes (et) pratiques - Entretien avec Isabelle Audinot et Sylvestre Meininger. Décadrages. Cinéma, à travers champs. Le doublage, 2324. Disponible sur : https://doi.org/10.4000/decadrages.695. DOI : 10.40000/decadrages.695.

Borowczyk, P. (2008). Les techniques adaptatrices utilisées par les traducteurs audiovisuels dans les versions doublées (française, polonaise et allemande) du film américain Shrek. In J. Lis, T. Tomaszkiewicz (eds.), Francophonie et interculturalité (pp. 13-22). Łask : Oficyna Wydawnicza LEKSEM.

Brisset, F. (2018). L'auteur de doublage, adaptateur du texte filmique : un traducteur-dialoguiste au service de la multimodalité. Studia Romanica Posnaniensia, 45/4, 23-45.

Brisset, F. (2017). Le doublage, à la frontière entre traduction et adaptation ? TranscUlturAl, 9.2. Disponible sur : https://journals.library.ualberta.ca/tc/index.php/TC/article/view/29339/21424.

Chaume, F. (2012). Audiovisual Translation : Dubbing. Manchester, UK \& Kinderhook (NY), USA : St. Jerome Publishing.

Ducrot, O., Schaeffer, J.-M. (1995). Nouveau dictionnaire encyclopédique des sciences du langage. Paris : Éditions du Seuil.

Guidère, M. (2010). Introduction à la traductologie. Penser la traduction : hier, aujourd'hui, demain. Bruxelles : De Boeck.

Joly, M. (2004). Introduction à l'analyse de l'image. Paris : Nathan/Sejer.

Lambert, J. \& Delabastita, D. (1996). La traduction de textes audiovisuels : modes et enjeux culturels. In Y. Gambier (ed.), Les transferts linguistiques dans les médias audiovisuels (pp. 33-58). Villeneuve d'Ascq (Nord) : Presses Universitaires du Septentrion.

Le Nouvel, T. (2007). Le doublage. Paris : Eyrolles.

Nord, Ch. (2008). La traduction : une activité ciblée. Introduction aux approches fonctionnalistes. Arras : Artois Presses Université.

Paquin, R. (2010). Le doublage synchrone ou entendre ce qu'on voit : Circuit. Le doublage, l'art de l'illusion, 107, 6-7.

Pettit, Z. (2008). Le sous-titrage: le rôle de l'image dans la traduction d'un texte multimodal. In J.-M. Lavaur, A. Şerban (eds.), La traduction audiovisuelle. Approche interdisciplinaire du sous-titrage (pp. 101-111). Bruxelles : De Boeck.

Plewa, E. (2015). Układy translacji audiowizualnych. In S. Grucza (ed.), Studia Naukowe 28. Warszawa : Wydawnictwo Naukowe Instytutu Komunikacji Specjalistycznej i Interkulturowej.

Sikora, I. (2013). Dubbing filmów animowanych. Strategie translatorskie w polskim dubbingu anglojęzycznych filmów animowanych. Nysa : Oficyna Wydawnicza PWSZ.

Tomaszkiewicz, T. (1999). Texte et image dans les communications aux masses. Poznań : Wydawnictwo Naukowe UAM.

\section{FILMOGRAPHIE}

Anderson, D.K. (prod.) \& Docter, P., Unkrich, L., Silverman, D. (dir.) (2001). Monsters, Inc. USA : Pixar Animation Studios.

Lipman, D., Williams, J.H., Warner, A. (prod.).\& Adamson, A., Vernon, C., Asbury, K. (dir.) (2004). Shrek 2. USA : DreamWorks.

Soria, M., Cheng, T. (prod.) \& Darnell, E., McGrath, T. (dir.) (2005). Madagascar. USA : DreamWorks. 